\title{
Search for signatures of magnetically-induced alignment in the arrival directions measured by the Pierre Auger Observatory
}

P. Abreu $^{\text {bl }}$, M. Aglietta ${ }^{\text {ay }}$, E.J. Ahn ${ }^{\text {ca }}$, I.F.M. Albuquerque ${ }^{\circ}$, D. Allard ${ }^{\text {ab }}$, I. Allekotte ${ }^{\mathrm{a}}$, J. Allen $^{\text {cd }}$, P. Allison $^{\text {cf }}$, J. Alvarez Castillo $^{\text {be }}$, J. Alvarez-Muñiz ${ }^{\text {bs }}$, M. Ambrosio ${ }^{\text {as }}$, A. Aminaei ${ }^{\text {bf }}$, L. Anchordoqui ${ }^{\mathrm{cm}}$, S. Andringa ${ }^{\text {bl }}$, T. Antičić w , A. Anzalone ax, C. Aramo as, E. Arganda bp, F. Arqueros bp , H. Asorey a , P. Assis ${ }^{\text {bl }}$, J. Aublin ad, M. Ave ${ }^{\mathrm{aj}}$, M. Avenier ${ }^{\mathrm{ae}}$, G. Avila ${ }^{\mathrm{j}}$, T. Bäcker ${ }^{\mathrm{an}}$, M. Balzer $^{\mathrm{a} i}$, K.B. Barber $^{\mathrm{k}}$, A.F. Barbosa $^{\mathrm{l}}$, R. Bardenet ${ }^{\mathrm{ac}}$, S.L.C. Barroso ${ }^{\text {r }}$, B. Baughman ${ }^{\text {cf }}$, J. Bäuml ${ }^{\text {ah }}$, J.J. Beatty ${ }^{\text {cf }}$, B.R. Becker ${ }^{\text {ck }}$, K.H. Becker ${ }^{\text {ag }}$, A. Bellétoile ${ }^{\text {af }}$, J.A. Bellido ${ }^{\mathrm{k}}$, S. BenZvi ${ }^{\mathrm{cl}}$, C. Berat ${ }^{\mathrm{ae}}$, X. Bertou ${ }^{\mathrm{a}}$, P.L. Biermann ${ }^{\text {ak }}$, P. Billoir ${ }^{\text {ad }}$, F. Blanco ${ }^{\text {bp }}$, M. Blanco $^{\text {bq }}$, C. Bleve ag, H. Blümer ${ }^{\text {aj,ah }}$, M. Boháčová ${ }^{y}$, D. Boncioli ${ }^{\text {at }}, C$. Bonifazi ${ }^{\text {u,ad }}$, R. Bonino $^{\text {ay }}$, N. Borodai $^{\text {bj }}$, J. Brack ${ }^{\text {by }}$, P. Brogueira bl, W.C. Brown ${ }^{\text {bz }}$, R. Bruijn ${ }^{\text {bu }}$, P. Buchholz ${ }^{\text {an }}$, A. Bueno ${ }^{\text {br }}$, R.E. Burton ${ }^{\text {bw }}$, K.S. Caballero-Mora ${ }^{\text {cg }}$, L. Caramete ${ }^{\mathrm{ak}}$, R. Caruso ${ }^{\mathrm{au}}$, A. Castellina ${ }^{\mathrm{ay}}$, O. Catalano $^{\mathrm{ax}}$, G. Cataldi $^{\text {ar }}$, L. Cazon ${ }^{\mathrm{bl}}$, R. Cester ${ }^{\mathrm{av}}$, J. Chauvin ${ }^{\mathrm{a}}$, S.H. Cheng ${ }^{\mathrm{cg}}$, A. Chiavassa ${ }^{\mathrm{ay}}$, J.A. Chinellato ${ }^{\mathrm{p}}$, A. Chou $^{\mathrm{ca}, \mathrm{cd}}$, J. Chudoba ${ }^{\mathrm{y}}$, R.W. Clay ${ }^{\mathrm{k}}$, M.R. Coluccia ${ }^{\mathrm{ar}}$, R. Conceição ${ }^{\mathrm{bl}}$, F. Contreras ${ }^{\mathrm{i}}$, H. Cook ${ }^{\text {bu }}$, M.J. Cooper ${ }^{\mathrm{k}}$, J. Coppens ${ }^{\text {bf,bh }}$, A. Cordier ${ }^{\text {ac }}$, S. Coutu ${ }^{c g}$, C.E. Covault ${ }^{\text {bw }}$, A. Creusot ${ }^{\text {ab,bn }}$, A. Criss ${ }^{c g}$, J. Cronin ${ }^{c i}$, A. Curutiu ${ }^{\text {ak }}$, S. Dagoret-Campagne ${ }^{\text {ac }}$, R. Dallier ${ }^{\text {af }}$, S. Dasso ${ }^{\text {f,d }}$, K. Daumiller $^{\text {ah }}$, B.R. Dawson ${ }^{\text {k }}$, R.M. de Almeida ${ }^{\text {, }}$, M. De Domenico ${ }^{\text {au }}$, C. De Donato ${ }^{\text {be,aq }}$, S.J. de Jong ${ }^{\text {bf,bh }}$, G. De La Vega ${ }^{\text {h }}$, W.J.M. de Mello Junior ${ }^{\mathrm{P}}$, J.R.T. de Mello Neto ${ }^{u}$, I. De Mitri ${ }^{\text {ar }}$, V. de Souza ${ }^{n}$, K.D. de Vries ${ }^{\text {bg }}$, G. Decerprit ${ }^{\text {ab }}$, L. del Peral ${ }^{\text {bq }}$, M. del Río ${ }^{\text {at,i }}$, O. Deligny ${ }^{\text {aa }}$, H. Dembinski ${ }^{\text {aj }}$, N. Dhital ${ }^{\text {cc }}$, C. Di Giulio ${ }^{\text {ap,at }}$, J.C. Diaz ${ }^{\text {cc }}$, M.L. Díaz Castro ${ }^{\mathrm{m}}$, P.N. Diep ${ }^{c n}$, C. Dobrigkeit ${ }^{\text {p }}$, W. Docters ${ }^{\text {bg }}$, J.C. D'Olivo ${ }^{\text {be }}$, P.N. Dong ${ }^{\text {cn,aa }}$, A. Dorofeev ${ }^{\text {by }}$, J.C. dos Anjos ${ }^{1}$,

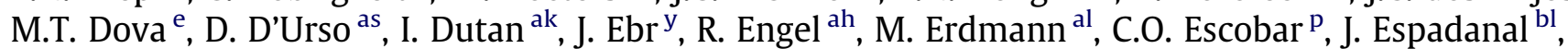
A. Etchegoyen ${ }^{\text {b }}$, P. Facal San Luis ${ }^{\text {ci }}$, I. Fajardo Tapia ${ }^{\text {be }}$, H. Falcke ${ }^{\text {bf,bi }}$, G. Farrar ${ }^{\text {cd }}$, A.C. Fauth ${ }^{\text {p }}$, N. Fazzini ${ }^{\text {ca }}$, A.P. Ferguson ${ }^{\text {bw }}$, A. Ferrero ${ }^{\text {b }}$, B. Fick ${ }^{c c}$, A. Filevich ${ }^{b}$, A. Filipčič ${ }^{\text {bm,bn }}$, S. Fliescher ${ }^{\text {al }}$, C.E. Fracchiolla ${ }^{\text {by }}$, E.D. Fraenkel ${ }^{\text {bg }}$, U. Fröhlich ${ }^{\text {an }}$, B. Fuchs ${ }^{1}$, R. Gaior ${ }^{\text {ad }}$, R.F. Gamarra ${ }^{b}$, S. Gambetta ${ }^{\text {ao }}$, B. García ${ }^{\text {h }}$, D. García Gámez ${ }^{\text {ac,br }}$, D. Garcia-Pinto ${ }^{\text {bp }}$, A. Gascon ${ }^{\text {br }}$, H. Gemmeke ${ }^{\text {ai }}$, K. Gesterling ${ }^{\text {ck }}$, P.L. Ghia ${ }^{\text {ad,ay }}$, U. Giaccari ${ }^{\text {ar }}$, M. Giller ${ }^{\text {bk }}$, H. Glass ${ }^{\text {ca }}$, M.S. Gold ${ }^{\text {ck }}$, G. Golup ${ }^{a}$, F. Gomez Albarracin ${ }^{\mathrm{e}}$, M. Gómez Berisso ${ }^{\mathrm{a}}$, P. Gonçalves ${ }^{\text {bl }}$, D. Gonzalez ${ }^{\text {aj }}$, J.G. Gonzalez ${ }^{\text {aj }}$, B. Gookin ${ }^{\text {by }}$, D. Góra aj,bj ${ }^{\text {A. Gorgi }}{ }^{\text {ay }}$, P. Gouffon ${ }^{\text {, }}$, S.R. Gozzini ${ }^{\text {bu }}$, E. Grashorn ${ }^{\text {cf }}$, S. Grebe ${ }^{\text {bf,bh }}$, N. Griffith ${ }^{\text {cf }}$, M. Grigat ${ }^{\text {al }}$, A.F. Grillo ${ }^{\text {az }}$, Y. Guardincerri ${ }^{\text {d }}$, F. Guarino ${ }^{\text {as }}$, G.P. Guedes q, A. Guzman ${ }^{\text {be }}$, J.D. Hague ${ }^{\text {ck }}$, P. Hansen e, D. Harari a , S. Harmsma ${ }^{\text {bg,bh }}$, J.L. Harton ${ }^{\text {by }}$, A. Haungs ${ }^{\text {ah }}$, T. Hebbeker ${ }^{\text {al }}$, D. Heck ${ }^{\text {ah }}$, A.E. Herve ${ }^{k}$, C. Hojvat ${ }^{\text {ca }}$, N. Hollon ${ }^{\text {ci }}$, V.C. Holmes ${ }^{k}$, P. Homola ${ }^{\text {bj }}$, J.R. Hörandel ${ }^{\text {bf }}$, A. Horneffer ${ }^{\text {bf }}$, M. Hrabovský ${ }^{z, y}$, T. Huege ${ }^{\text {ah }}$, A. Insolia ${ }^{\text {au }}$, F. Ionita ${ }^{\text {ci }}$, A. Italiano au , C. Jarne e, S. Jiraskova ${ }^{\text {bf }}$, M. Josebachuili ${ }^{\mathrm{b}}$, K. Kadija ${ }^{\text {w }}$, K.H. Kampert ${ }^{\text {ag }}$, P. Karhan ${ }^{\mathrm{x}}$, P. Kasper ${ }^{\text {ca }}$, B. Kégl ${ }^{\text {ac }}$, B. Keilhauer ${ }^{\text {ah }}$, A. Keivani ${ }^{\text {cb }}$, J.L. Kelley ${ }^{\text {bf }}$, E. Kemp ${ }^{\text {p }}$, R.M. Kieckhafer ${ }^{c c}$, H.O. Klages ${ }^{\text {ah }}$, M. Kleifges ${ }^{\text {ai }}$, J. Kleinfeller ${ }^{\text {ah }}$, J. Knapp ${ }^{\text {bu }}$, D.-H. Koang ${ }^{\text {ae }}$, K. Kotera ${ }^{\text {ci }}$, N. Krohm ${ }^{\text {ag }}$, O. Krömer ${ }^{\text {ai }}$, D. Kruppke-Hansen ${ }^{\text {ag }}$, F. Kuehn ${ }^{\text {ca }}$, D. Kuempel ${ }^{\text {ag }}$, J.K. Kulbartz ${ }^{\text {am }}$, N. Kunka ${ }^{\text {ai }}$, G. La Rosa ${ }^{\text {ax }}$, ${\text { C. } \text { Lachaud }^{\mathrm{ab}} \text {, P. Lautridou }}^{\text {af }}$, M.S.A.B. Leão ${ }^{\mathrm{t}}$, D. Lebrun ${ }^{\mathrm{ae}}$, P. Lebrun ${ }^{\mathrm{ca}}$, M.A. Leigui de Oliveira ${ }^{\mathrm{t}}$, A. Lemiere ${ }^{\text {aa }}$, A. Letessier-Selvon ${ }^{\text {ad }}$, I. Lhenry-Yvon ${ }^{\text {aa }}$, K. Link $^{\text {aj }}$, R. López ${ }^{\text {bb }}$, A. Lopez Agüera ${ }^{\text {bs }}$, K. Louedec ${ }^{\text {ac }}$, J. Lozano Bahilo ${ }^{\text {br }}$, L. Lu ${ }^{\text {bu }}$, A. Lucero ${ }^{\text {bay }}$, M. Ludwig ${ }^{\text {aj }}$, H. Lyberis ${ }^{\text {aa }}$, M.C. Maccarone ${ }^{\text {ax }}$, C. Macolino $^{\text {ad }}$, S. Maldera ${ }^{\text {ay }}$, D. Mandat ${ }^{y}$, P. Mantsch ${ }^{\text {ca }}$, A.G. Mariazzi e, J. Marin i,ay, V. Marin ${ }^{\text {af }}$, I.C. Maris ${ }^{\text {ad }}$, H.R. Marquez Falcon ${ }^{\text {bd }}$, G. Marsella ${ }^{\text {aw }}$, D. Martello ${ }^{\text {ar }}$, L. Martin ${ }^{\text {af }}$, H. Martinez ${ }^{\text {bc }}$, O. Martínez Bravo ${ }^{\text {bb }}$, H.J. Mathes ${ }^{\text {ah }}$, J. Matthews ${ }^{\text {cb,ch }}$, J.A.J. Matthews ${ }^{\text {ck }}$, G. Matthiae ${ }^{\text {at }}$, D. Maurizio ${ }^{\text {av }}$, P.O. Mazur ${ }^{\text {ca }}$, G. Medina-Tanco ${ }^{\text {be }}$, M. Melissas ${ }^{\text {aj }}$, D. Melo ${ }^{\text {b,av }}$, E. Menichetti ${ }^{\text {av }}$, A. Menshikov ${ }^{\text {ai }}$, 
P. Mertsch ${ }^{\text {bt }}$, C. Meurer ${ }^{\text {al }}$, S. Mićanović ${ }^{\text {w }}$, M.I. Micheletti ${ }^{g}$, W. Miller ${ }^{\text {ck }}$, L. Miramonti ${ }^{\text {aq }}$,

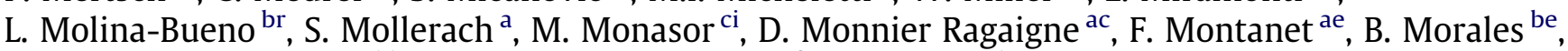

C. Morello $^{\text {ay }}$, E. Moreno ${ }^{\text {bb }}$, J.C. Moreno ${ }^{\text {e }}$, C. Morris ${ }^{\text {cf }}$, M. Mostafá by , C.A. Moura ${ }^{\text {t,as }}$,

S. Mueller ${ }^{\text {ah }}$, M.A. Muller ${ }^{\mathrm{p}}$, G. Müller ${ }^{\text {al }}$, M. Münchmeyer ${ }^{\text {ad }}$, R. Mussa ${ }^{\text {av }}$, G. Navarra ${ }^{\text {ay, } 1}$, J.L. Navarro ${ }^{\text {br }}$,

S. Navas ${ }^{\text {br }}$, P. Necesal ${ }^{\mathrm{y}}$, L. Nellen ${ }^{\text {be }}$, A. Nelles ${ }^{\text {bf,bh }}$, J. Neuser ${ }^{\text {ag }}$, P.T. Nhung ${ }^{\text {cn }}$, L. Niemietz $^{\text {ag }}$,

N. Nierstenhoefer ${ }^{\mathrm{ag}}$, D. Nitz ${ }^{\mathrm{cc}}, \mathrm{D}$. Nosek ${ }^{\mathrm{x}}$, L. Nožka ${ }^{\mathrm{y}}$, M. Nyklicek ${ }^{\mathrm{y}}$, J. Oehlschläger ${ }^{\mathrm{ah}}$, A. Olinto ${ }^{\mathrm{ci}}$,

P. Oliva ${ }^{\text {ag }}$, V.M. Olmos-Gilbaja ${ }^{\text {bs }}$, M. Ortiz ${ }^{\text {bp }}$, N. Pacheco ${ }^{\text {bq }}$, D. Pakk Selmi-Dei ${ }^{\text {p }}$, M. Palatka ${ }^{\mathrm{y}}$, J. Pallotta $^{\mathrm{c}}$,

N. Palmieri ${ }^{\text {aj }}$, G. Parente ${ }^{\text {bs }}$, E. Parizot ${ }^{\text {ab }}$, A. Parra ${ }^{\text {bs }}$, R.D. Parsons ${ }^{\text {bu }}$, S. Pastor ${ }^{\text {bo }}$, T. Paul $^{\text {ce }}$, M. Pech $^{\mathrm{y}}$,

J. Pȩkala ${ }^{\text {bj }}$, R. Pelayo ${ }^{\text {bs }}$, I.M. Pepe ${ }^{\mathrm{s}}$, L. Perrone ${ }^{\mathrm{aw}}$, R. Pesce ${ }^{\mathrm{ao}}$, E. Petermann ${ }^{\mathrm{cj}}$, S. Petrera ${ }^{\text {ap }}$, P. Petrinca ${ }^{\text {at }}$,

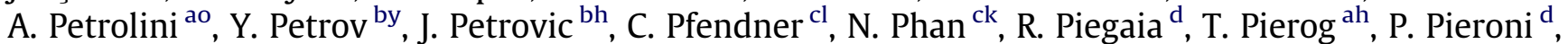

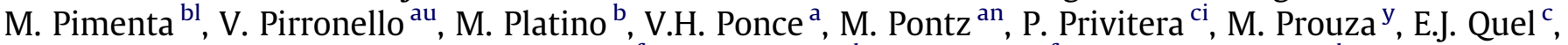

S. Querchfeld ${ }^{\text {ag }}$, J. Rautenberg ${ }^{\text {ag }}$, O. Ravel $^{\text {af }}$, D. Ravignani ${ }^{\text {b }}$, B. Revenu ${ }^{\text {af }}$, J. Ridky ${ }^{\text {, }}$, S. Riggi ${ }^{\text {bs,au }}{ }^{\text {, M. Risse }}{ }^{\text {an }}$,

P. Ristori ${ }^{c}$, H. Rivera ${ }^{\text {aq }}$, V. Rizi ${ }^{\text {ap }}$, J. Roberts ${ }^{c d}$, C. Robledo ${ }^{\text {bb }}$, W. Rodrigues de Carvalho ${ }^{\text {bs,o }}$, G. Rodriguez ${ }^{\text {bs }}$,

J. Rodriguez Martino ${ }^{\text {i }, ~ J . ~ R o d r i g u e z ~ R o j o ~}{ }^{\text {i }}$, I. Rodriguez-Cabo ${ }^{\text {bs }}$, M.D. Rodríguez-Frías ${ }^{\text {bq }}$, G. Ros ${ }^{\text {bq }}$,

J. Rosado $^{\text {bp }}$, T. Rossler ${ }^{z}$, M. Roth ${ }^{\text {ah }}$, B. Rouillé-d'Orfeuil ${ }^{\text {ci }}$, E. Roulet ${ }^{\text {a }}$, A.C. Rovero ${ }^{\text {f }}$, C. Rühle ${ }^{\text {ai }}$,

F. Salamida ap,ah, H. Salazar ${ }^{\text {bb }}$, G. Salina ${ }^{\text {at }}$, F. Sánchez ${ }^{\text {b }}$, C.E. Santo ${ }^{\text {bl }}$, E. Santos ${ }^{\text {bl }}$, E.M. Santos ${ }^{\text {u }}$,

F. Sarazin ${ }^{\text {bx }}$, B. Sarkar ${ }^{\text {ag }}$, S. Sarkar ${ }^{\text {bt }}$, R. Sato ${ }^{i}$, N. Scharf ${ }^{\text {al }}$, V. Scherini ${ }^{\text {aq }}$, H. Schieler ${ }^{\text {ah }}$, P. Schiffer ${ }^{\text {al }}$,

A. Schmidt ${ }^{\text {ai }}$, F. Schmidt ${ }^{\text {ci }}$, O. Scholten ${ }^{\text {bg }}$, H. Schoorlemmer ${ }^{\text {bf,bh }}$, J. Schovancova ${ }^{\mathrm{y}}$, P. Schovánek ${ }^{\mathrm{y}}$,

F. Schröder ${ }^{\text {ah }}$, S. Schulte ${ }^{\text {al }}$, D. Schuster ${ }^{\text {bx }}$, S.J. Sciutto ${ }^{\text {e }}$, M. Scuderi ${ }^{\text {au }}$, A. Segreto $^{\text {ax }}$, M. Settimo ${ }^{\text {an }}$,

A. Shadkam ${ }^{\text {cb }}$, R.C. Shellard ${ }^{1, m}$, I. Sidelnik ${ }^{\text {b }, ~ G . ~ S i g l ~ a m ~, ~ H . H . ~ S i l v a ~ L o p e z ~}{ }^{\text {be }}$, A. Śmiałkowski ${ }^{\text {bk }}$, R. Šmída ah,y,

G.R. Snow ${ }^{\text {cj }}$, P. Sommers ${ }^{\text {cg }}$, J. Sorokin ${ }^{k}$, H. Spinka ${ }^{\text {bv,ca }}{ }^{\text {, R. Squartini }}{ }^{\text {i }}$, S. Stanic ${ }^{\text {bn }}$, J. Stapleton ${ }^{\text {cf }}$,

J. Stasielak $^{\text {bj }}$, M. Stephan ${ }^{\text {al }}$, E. Strazzeri ${ }^{\text {ax }}$, A. Stutz $^{\text {ae }}$, F. Suarez ${ }^{\text {b }}$, T. Suomijärvi ${ }^{\text {aa }}$, A.D. Supanitsky ${ }^{\text {f,be }}$,

T. Šuša ${ }^{\text {w }}$, M.S. Sutherland ${ }^{\text {cb,cf, J. Swain }}{ }^{\text {ce }}$, Z. Szadkowski ${ }^{\text {bk,ag }}$, M. Szuba ah, A. Tamashirof, A. Tapia ${ }^{\text {b }}$,

M. Tartare ae ${ }^{\text {a }}$ O. Taşcău ${ }^{\text {ag }}$, C.G. Tavera Ruiz ${ }^{\text {be }}$, R. Tcaciuc ${ }^{\text {an }}$, D. Tegolo ${ }^{\text {au,ba }}{ }^{\text {, N.T. Thao }}{ }^{\text {cn }}$, D. Thomas ${ }^{\text {by }}$, J. Tiffenberg ${ }^{\mathrm{d}}$, C. Timmermans ${ }^{\text {bh,bf }}$, D.K. Tiwari ${ }^{\text {bd }}$, W. Tkaczyk ${ }^{\text {bk }}$, C.J. Todero Peixoto $^{\text {n,t }}$, B. Tomé ${ }^{\text {bl }}$,

A. Tonachini ${ }^{a v}$, P. Travnicek ${ }^{\mathrm{y}}$, D.B. Tridapalli ${ }^{\mathrm{o}}$, G. Tristram $^{\mathrm{ab}}, \mathrm{E}$. Trovato ${ }^{\mathrm{au}}$, M. Tueros $^{\mathrm{bs}, \mathrm{d}}$, R. Ulrich $^{\mathrm{cg}, \mathrm{ah}}$,

M. Unger ${ }^{\text {ah }}$, M. Urban ac , J.F. Valdés Galicia be, I. Valiño bs,ah, L. Valore ${ }^{\text {as }}$, A.M. van den Berg bg, E. Varela ${ }^{\text {bb }}$,

B. Vargas Cárdenas ${ }^{\text {be }}$, J.R. Vázquez ${ }^{\text {bp }}$, R.A. Vázquez ${ }^{\text {bs }}$, D. Veberič ${ }^{\text {bn,bm }}{ }^{\text {, V. Verzi }}{ }^{\text {at }}$, J. Vicha $^{\mathrm{y}}$, M. Videla $^{\text {h, }}$,

L. Villaseñor $^{\text {bd }}$, H. Wahlberg ${ }^{\mathrm{e}}$, P. Wahrlich ${ }^{\mathrm{k}}$, O. Wainberg ${ }^{\mathrm{b}}$, D. Walz ${ }^{\mathrm{al}}$, D. Warner ${ }^{\text {by }}$, A.A. Watson ${ }^{\text {bu }}$,

M. Weber ${ }^{\text {ai }}$, K. Weidenhaupt ${ }^{\text {al }}$, A. Weindl ${ }^{\text {ah }}$, S. Westerhoff ${ }^{c l}$, B.J. Whelan ${ }^{k}$, G. Wieczorek ${ }^{\text {bk }}$, L. Wiencke $^{\text {bx }}$,

B. Wilczyńska ${ }^{\text {bj }}$, H. Wilczyński ${ }^{\text {bj }}$, M. Will ${ }^{\text {ah }}$, C. Williams ${ }^{\text {ci }}$, T. Winchen ${ }^{\text {al }}$, M.G. Winnick ${ }^{\mathrm{k}}$, M. Wommer $^{\text {ah }}$,

B. Wundheiler ${ }^{b}$, T. Yamamoto ${ }^{\text {ci,2 }}$, T. Yapici ${ }^{\text {cc }}$, P. Younk ${ }^{\text {an }}$, G. Yuan $^{c b}$, A. Yushkov ${ }^{\text {bs,as }}$, B. Zamorano ${ }^{\text {br }}$,

E. Zas ${ }^{\text {bs }}$, D. Zavrtanik ${ }^{\text {bn,bm }}$, M. Zavrtanik ${ }^{\text {bm,bn }}$, I. Zaw ${ }^{\text {cd }}$, A. Zepeda ${ }^{\text {bc }}$, M. Zimbres Silva ${ }^{\text {ag,p, }}$

M. Ziolkowski an , The Pierre Auger Collaboration

\footnotetext{
${ }^{a}$ Centro Atómico Bariloche and Instituto Balseiro (CNEA-UNCuyo-CONICET), San Carlos de Bariloche, Argentina

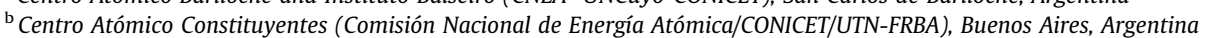

c Centro de Investigaciones en Láseres y Aplicaciones, CITEFA and CONICET, Argentina

${ }^{\mathrm{d}}$ Departamento de Física, FCEyN, Universidad de Buenos Aires y CONICET, Argentina

e IFLP, Universidad Nacional de La Plata and CONICET, La Plata, Argentina

${ }^{\mathrm{f}}$ Instituto de Astronomía y Física del Espacio (CONICET-UBA), Buenos Aires, Argentina

${ }^{\mathrm{g}}$ Instituto de Física de Rosario (IFIR) - CONICET/U.N.R. and Facultad de Ciencias Bioquímicas y Farmacéuticas U.N.R., Rosario, Argentina

${ }^{\mathrm{h}}$ National Technological University, Faculty Mendoza (CONICET/CNEA), Mendoza, Argentina

${ }^{\mathrm{i}}$ Pierre Auger Southern Observatory, Malargüe, Argentina

${ }^{j}$ Pierre Auger Southern Observatory and Comisión Nacional de Energía Atómica, Malargüe, Argentina

${ }^{\mathrm{k}}$ University of Adelaide, Adelaide, S.A., Australia

${ }^{1}$ Centro Brasileiro de Pesquisas Fisicas, Rio de Janeiro, RJ, Brazil

${ }^{\mathrm{m}}$ Pontifícia Universidade Católica, Rio de Janeiro, RJ, Brazil

${ }^{\mathrm{n}}$ Universidade de São Paulo, Instituto de Física, São Carlos, SP, Brazil

${ }^{\circ}$ Universidade de São Paulo, Instituto de Física, São Paulo, SP, Brazil

${ }^{\mathrm{P}}$ Universidade Estadual de Campinas, IFGW, Campinas, SP, Brazil

${ }^{\mathrm{q}}$ Universidade Estadual de Feira de Santana, Brazil

${ }^{\mathrm{r}}$ Universidade Estadual do Sudoeste da Bahia, Vitoria da Conquista, BA, Brazil

${ }^{s}$ Universidade Federal da Bahia, Salvador, BA, Brazil

${ }^{\mathrm{t}}$ Universidade Federal do ABC, Santo André, SP, Brazil

${ }^{\mathrm{u}}$ Universidade Federal do Rio de Janeiro, Instituto de Física, Rio de Janeiro, RJ, Brazil

${ }^{\mathrm{v}}$ Universidade Federal Fluminense, EEIMVR, Volta Redonda, RJ, Brazil

${ }^{\mathrm{w}}$ Rudjer Bošković Institute, 10000 Zagreb, Croatia

${ }^{\mathrm{x}}$ Charles University, Faculty of Mathematics and Physics, Institute of Particle and Nuclear Physics, Prague, Czech Republic

${ }^{\mathrm{y}}$ Institute of Physics of the Academy of Sciences of the Czech Republic, Prague, Czech Republic

${ }^{\mathrm{z}}$ Palacky University, RCATM, Olomouc, Czech Republic

aa Institut de Physique Nucléaire d'Orsay (IPNO), Université Paris 11, CNRS-IN2P3, Orsay, France
} 
${ }^{\mathrm{ab}}$ Laboratoire AstroParticule et Cosmologie (APC), Université Paris 7, CNRS-IN2P3, Paris, France

${ }^{a c}$ Laboratoire de l'Accélérateur Linéaire (LAL), Université Paris 11, CNRS-IN2P3, Orsay, France

${ }^{\text {ad }}$ Laboratoire de Physique Nucléaire et de Hautes Energies (LPNHE), Universités Paris 6 et Paris 7, CNRS-IN2P3, Paris, France

ae Laboratoire de Physique Subatomique et de Cosmologie (LPSC), Université Joseph Fourier, INPG, CNRS-IN2P3, Grenoble, France

af SUBATECH, École des Mines de Nantes, CNRS-IN2P3, Université de Nantes, Nantes, France

${ }^{\mathrm{ag}}$ Bergische Universität Wuppertal, Wuppertal, Germany

${ }^{\text {ah }}$ Karlsruhe Institute of Technology, Campus North, Institut für Kernphysik, Karlsruhe, Germany

ai Karlsruhe Institute of Technology, Campus North, Institut für Prozessdatenverarbeitung und Elektronik, Karlsruhe, Germany

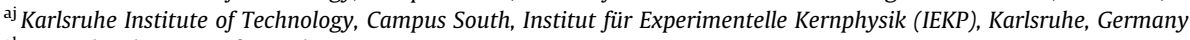

${ }^{\mathrm{ak}}$ Max-Planck-Institut für Radioastronomie, Bonn, Germany

${ }^{\text {al }}$ RWTH Aachen University, III, Physikalisches Institut A, Aachen, Germany

am Universität Hamburg, Hamburg, Germany

an Universität Siegen, Siegen, Germany

ao Dipartimento di Fisica dell'Università and INFN, Genova, Italy

${ }^{a p}$ Università dell'Aquila and INFN, L'Aquila, Italy

${ }^{\mathrm{aq}}$ Università di Milano and Sezione INFN, Milan, Italy

${ }^{\text {ar }}$ Dipartimento di Fisica dell'Università del Salento and Sezione INFN, Lecce, Italy

as Università di Napoli "Federico II" and Sezione INFN, Napoli, Italy

at Università di Roma II "Tor Vergata” and Sezione INFN, Roma, Italy

au Università di Catania and Sezione INFN, Catania, Italy

${ }^{a v}$ Università di Torino and Sezione INFN, Torino, Italy

${ }^{\mathrm{aw}}$ Dipartimento di Ingegneria dell'Innovazione dell'Università del Salento and Sezione INFN, Lecce, Italy

${ }^{a x}$ Istituto di Astrofisica Spaziale e Fisica Cosmica di Palermo (INAF), Palermo, Italy

ay Istituto di Fisica dello Spazio Interplanetario (INAF), Università di Torino and Sezione INFN, Torino, Italy

az INFN, Laboratori Nazionali del Gran Sasso, Assergi (L'Aquila), Italy

ba Università di Palermo and Sezione INFN, Catania, Italy

${ }^{\mathrm{bb}}$ Benemérita Universidad Autónoma de Puebla, Puebla, Mexico

bc Centro de Investigación y de Estudios Avanzados del IPN (CINVESTAV), México, D.F., Mexico

${ }^{\text {bd }}$ Universidad Michoacana de San Nicolas de Hidalgo, Morelia, Michoacan, Mexico

be Universidad Nacional Autonoma de Mexico, Mexico, D.F., Mexico

${ }^{b f}$ IMAPP, Radboud University Nijmegen, Netherlands

bg Kernfysisch Versneller Instituut, University of Groningen, Groningen, Netherlands

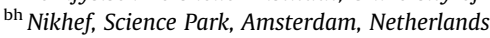

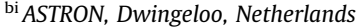

${ }^{b j}$ Institute of Nuclear Physics PAN, Krakow, Poland

bk University of Łódź, Łódź, Poland

${ }^{\mathrm{bl}}$ LIP and Instituto Superior Técnico, Technical University of Lisbon, Portugal

${ }^{\mathrm{bm}}$ J. Stefan Institute, Ljubljana, Slovenia

bn Laboratory for Astroparticle Physics, University of Nova Gorica, Slovenia

bo Instituto de Física Corpuscular, CSIC-Universitat de València, Valencia, Spain

${ }^{\mathrm{bp}}$ Universidad Complutense de Madrid, Madrid, Spain

${ }^{\mathrm{bq}}$ Universidad de Alcalá, Alcalá de Henares (Madrid), Spain

${ }^{\mathrm{br}}$ Universidad de Granada \& C.A.F.P.E., Granada, Spain

bs Universidad de Santiago de Compostela, Spain

${ }^{\text {bt }}$ Rudolf Peierls Centre for Theoretical Physics, University of Oxford, Oxford, United Kingdom

${ }^{\text {bu }}$ School of Physics and Astronomy, University of Leeds, United Kingdom

${ }^{\text {bv }}$ Argonne National Laboratory, Argonne, IL, USA

bw Case Western Reserve University, Cleveland, OH, USA

${ }^{\mathrm{bx}}$ Colorado School of Mines, Golden, CO, USA

by Colorado State University, Fort Collins, CO, USA

${ }^{\mathrm{bz}}$ Colorado State University, Pueblo, CO, USA

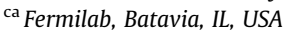

${ }^{\mathrm{cb}}$ Louisiana State University, Baton Rouge, LA, USA

${ }^{\mathrm{cc}}$ Michigan Technological University, Houghton, MI, USA

${ }^{\mathrm{cd}}$ New York University, New York, NY, USA

${ }^{c e}$ Northeastern University, Boston, MA, USA

${ }^{\mathrm{cf}}$ Ohio State University, Columbus, $\mathrm{OH}$, USA

${ }^{c g}$ Pennsylvania State University, University Park, PA, USA

${ }^{c h}$ Southern University, Baton Rouge, LA, USA

ci University of Chicago, Enrico Fermi Institute, Chicago, IL, USA

${ }^{c j}$ University of Nebraska, Lincoln, NE, USA

${ }^{\mathrm{ck}}$ University of New Mexico, Albuquerque, NM, USA

${ }^{\mathrm{cl}}$ University of Wisconsin, Madison, WI, USA

${ }^{\mathrm{cm}}$ University of Wisconsin, Milwaukee, WI, USA

${ }^{\mathrm{cn}}$ Institute for Nuclear Science and Technology (INST), Hanoi, Viet nam

\section{A R T I C L E I N F O}

\section{Article history:}

Received 22 July 2011

Received in revised form 26 September 2011

Accepted 7 October 2011

Available online 18 October 2011

\section{A B S T R A C T}

We present the results of an analysis of data recorded at the Pierre Auger Observatory in which we search for groups of directionally-aligned events (or 'multiplets') which exhibit a correlation between arrival direction and the inverse of the energy. These signatures are expected from sets of events coming from the same source after having been deflected by intervening coherent magnetic fields. The observation of several

E-mail address: auger_spokepersons@fnal.gov

\footnotetext{
1 Deceased.

2 At Konan University, Kobe, Japan.
} 
Keywords:

Ultra-high energy cosmic rays

Pierre auger observatory

Arrival directions events from the same source would open the possibility to accurately reconstruct the position of the source and also measure the integral of the component of the magnetic field orthogonal to the trajectory of the cosmic rays. We describe the largest multiplets found and compute the probability that they appeared by chance from an isotropic distribution. We find no statistically significant evidence for the presence of multiplets arising from magnetic deflections in the present data.

\section{Introduction}

The origin of ultra-high energy cosmic rays is a long-standing open question, and the identification of their sources is one of the primary motivations for the research conducted at the Pierre Auger Observatory. If the density of cosmic rays sources is not too large, it is expected that there could be indications of the presence of multiplets, i.e. sets of events with different energy that come from a single point-like source. Due to the magnetic fields that cosmic rays traverse on their paths from their sources to the Earth, they will be deflected and this deflection is proportional to the inverse of their energy if the deflections are small. Therefore, to identify sets of cosmic rays that come from a single source, a search for events that show a correlation between their arrival direction and the inverse of their energy has been performed using the data recorded at the Pierre Auger Observatory. The observation of cosmic ray multiplets could allow for the accurate location of the direction of the source and could also provide a new means to probe the galactic magnetic field, as it should be possible to infer the value of the integral of the component of the magnetic field orthogonal to the trajectory of the cosmic rays. Note that to observe a correlated multiplet the source should be steady, in the sense that its lifetime is larger than the difference in the time delays due to the propagation in the intervening magnetic fields for the energies considered. Moreover, magnetic fields should also be steady in the same sense so that cosmic rays traverse approximately the same fields.

This study relies on the acceleration at the source of a proton component (or intermediate mass nuclei being accelerated and photo-disintegrated during extragalactic propagation with the deflections due to extragalactic magnetic fields being small compared to those in the Galaxy). Due to the magnitude of the known magnetic fields involved, heavy nuclei at these energies would appear spread over a very large region of the sky, probing regions with different amplitudes and directions of the magnetic field, and hence losing their alignment and correlation with the inverse of energy.

The galactic magnetic field is poorly constrained by the available data, even though there has been considerable effort to improve this knowledge using different observational techniques, see, e.g. [1-3]. This field is usually described as the superposition of a large-scale regular component and a turbulent one. The regular component has a few $\mu \mathrm{G}$ strength and is coherent on scales of a few kpc with a structure related to the spiral arms of the galactic disk, and eventually also a more extended halo component (see, e.g. [4]). The deflection of cosmic rays with energy $E$ and charge $Z$ by the regular component of the magnetic field $\vec{B}$ after traversing a distance $L$ is given by

$\delta \simeq 16^{\circ} \frac{20 \mathrm{EeV}}{E / Z}\left|\int_{0}^{L} \frac{\mathrm{d} \vec{l}}{3 \mathrm{kpc}} \times \frac{\overrightarrow{\mathrm{B}}}{2 \mu \mathrm{G}}\right|$,

where $1 \mathrm{EeV} \equiv 10^{18} \mathrm{eV}$. This is the predominant deflection because, although the turbulent component has a root mean square amplitude of $B_{\text {rms }} \simeq(1-2) B_{\text {reg, }}$ it has a much smaller coherence length (typically $L_{c} \simeq 50-100 \mathrm{pc}$ ) [5,6], leading to a smaller deflection, with a typical root mean square value $\delta_{\mathrm{rms}} \simeq 1.5^{\circ} \frac{20 \mathrm{EeV}}{E / Z} \frac{\mathrm{B}_{\mathrm{rms}}}{3 \mu \mathrm{G}} \sqrt{\frac{L}{1 \mathrm{kpc}}} \sqrt{\frac{L_{c}}{50 \mathrm{pc}}}$.

After traveling a distance $L$ through the turbulent field, the trajectories of cosmic rays would be displaced a distance $\sim \delta_{\text {rms }} L$ with respect to the one they would have had if only the regular field were present. If this displacement is smaller than the coherence length $L_{c}$, this means that all the particles with that energy have experienced nearly the same values of the turbulent field along their trajectories. Thus, the effect is that the arrival direction of cosmic rays will coherently wiggle with an amplitude $\delta_{\text {rms }}(E)$ around the direction determined by the deflection due to the regular magnetic field as a function of the energy. Conversely, when $\delta_{\text {rms }}(E) L>L_{c}$, particles of the same energy that have probed uncorrelated values of the turbulent field are able to reach the observer from the source and several images appear, scattered by $\delta_{\text {rms }}(E)$ around the image that would be produced by the regular field alone. Which of the two regimes actually takes place depends on the energy considered and on the distance traveled in the turbulent field. For instance, for $L \simeq 2 \mathrm{kpc}$ and energy about $20 \mathrm{EeV}$, the second situation applies, while at much higher energies the first one holds.

Extragalactic magnetic fields could also deflect the trajectories of cosmic rays, but their strength is yet unknown and the relevance of their effect is a matter of debate, see, e.g. [7-9].

\section{The Pierre Auger Observatory and the data set}

The Pierre Auger Observatory, located in Malargüe, Argentina $\left(35.2^{\circ} \mathrm{S}, 69.5^{\circ} \mathrm{W}\right)$ at $1400 \mathrm{~m}$ a.s.l. [10], was designed to measure ultra-high energy cosmic rays (energy $E>10^{18}$ ) with unprecedented statistics. It consists of a surface array of 1660 water-Cherenkov stations. The surface array is arranged in an equilateral triangular grid with $1500 \mathrm{~m}$ spacing, covering an area of approximately $3000 \mathrm{~km}^{2}$ [11]. The array is overlooked by 27 fluorescence telescopes located on hills at four sites on its periphery [12]. The surface and air fluorescence detectors are designed to perform complementary measurements of air showers created by cosmic rays. The surface array is used to observe the lateral distribution of the air shower particles at ground level, while the fluorescence telescopes are used to record the longitudinal development of the shower as it moves through the atmosphere.

In this work we analyze events with zenith angles smaller than $60^{\circ}$ recorded by the surface detector from 1 st January 2004 to 31st December 2010. The events are required to have at least five active stations surrounding the station with the highest signal, and the reconstructed core must be inside an active equilateral triangle of stations [13]. The corresponding exposure is $25,806 \mathrm{~km}^{2} \mathrm{sr} \mathrm{yr}$. The angular resolution, defined as the 68th percentile of the distribution of opening angles between the true and reconstructed directions of simulated events, is better than $0.9^{\circ}$ for events that trigger at least six surface stations $(E>10 \mathrm{EeV})$ [14]. The energy resolution is about $15 \%$ and the absolute energy scale, given by the fluorescence calibration, has a systematic uncertainty of $22 \%$ [15]. 


\section{Method adopted for the multiplets search}

In the limit of large energy, and hence small deflections, it is a good approximation to consider the following simplified relation between the cosmic ray observed arrival directions, described by the unit vector $\vec{\theta}$, and the actual source direction $\overrightarrow{\theta_{s}}$

$\vec{\theta}=\overrightarrow{\theta_{s}}+\frac{Z e}{E} \int_{0}^{L} \mathrm{~d} \vec{l} \times \vec{B} \simeq \overrightarrow{\theta_{s}}+\frac{\vec{D}\left(\overrightarrow{\theta_{s}}\right)}{E}$

where Ze is the electric charge of the cosmic ray and $D \equiv\left|\vec{D}\left(\overrightarrow{\theta_{s}}\right)\right|$ will be called the deflection power and will be given in units of $1^{\circ}$ $100 \mathrm{EeV}$, which is $\approx 1.9$ e $\mu \mathrm{G} \mathrm{kpc}$.

In the case of proton sources, departures from the linear approximation are relevant for energies below $20 \mathrm{EeV}$ for typical galactic magnetic field models [16], as the deflections of the trajectories are large and the integral of the magnetic field component orthogonal to the path cannot be approximated as a constant for a fixed source direction. This fact motivates the restriction of the present analysis to events with energies above $20 \mathrm{EeV}$.

In order to identify sets of events coming from the same source, the main requirement will be that they appear aligned in the sky and have a high value of the correlation coefficient between the arrival direction and the inverse of the energy.

To compute the correlation coefficient for a given subset of $N$ nearby event directions, we first identify the axis along which the correlation is maximal. For this we initially use an arbitrary coordinate system $(x, y)$ in the tangent plane to the celestial sphere (centered in the average direction to the events) and compute the covariance

$\operatorname{Cov}(x, 1 / E)=\frac{1}{N} \sum_{i=1}^{N}\left(x_{i}-\langle x\rangle\right)\left(1 / E_{i}-\langle 1 / E\rangle\right)$

and similarly for $\operatorname{Cov}(y, 1 / E)$. We then rotate the coordinates to a system $(u, w)$ in which $\operatorname{Cov}(w, 1 / E)=0$, and hence $\operatorname{Cov}(u, 1 / E)$ is maximal. This corresponds to a rotation angle between the $u$ and $x$ axes given by

$\alpha=\arctan \left(\frac{\operatorname{Cov}(y, 1 / E)}{\operatorname{Cov}(x, 1 / E)}\right)$.

The correlation between $u$ and $1 / E$ is measured through the correlation coefficient

$C(u, 1 / E)=\frac{\operatorname{Cov}(u, 1 / E)}{\sqrt{\operatorname{Var}(u) \operatorname{Var}(1 / E)}}$,

where the variances are given by $\operatorname{Var}(x)=\left\langle(x-\langle x\rangle)^{2}\right\rangle$. We demonstrate this procedure in Fig. 1. In the left panel we show the selection of coordinates $u$ and $w$ for a set of events of a simulated source superimposed on a background of isotropically distributed events. In the right panel the correlation between $u$ and $1 / E$ for the same source events is plotted.

A given set of events will be identified as a correlated multiplet when $C(u, 1 / E)>C_{\min }$ and, when the spread in the transverse direction $w$ is small, $W=\max \left(\left|w_{i}-\langle w\rangle\right|\right)<W_{\max }$ (corresponding to a total width of $\sim 2 W_{\max }$ in the perpendicular direction). The values for $C_{\min }$ and $W_{\max }$ were chosen as a compromise between maximizing the signal from a true source and minimizing the background arising from chance alignments. In order to determine the optimal values of these quantities, we performed numerical simulations of sets of events from randomly-located extragalactic sources. In these simulations, protons were propagated through a bisymmetric magnetic field with even symmetry (BSS-S) $[17,18]$ (the local value of the field used was $2 \mu \mathrm{G}$ ) and the effect of the turbulent magnetic field was included by simply adding a random deflection with root mean square amplitude $\delta_{\text {rms }}=1.5^{\circ}(20 \mathrm{EeV} / E)$. Although the latter is a rough approximation, and a dependence on the arrival directions should be expected, it is good enough for the purpose of fixing $C_{\min }$ and $W_{\max }$. We considered one hundred extragalactic sources located at random isotropic directions and simulated sets of $N$ events coming from each source $(N=14,13,12)$. The energy of the events followed an $E^{-2}$ spectrum at the source and we added random gaussian uncertainties in the angular directions and energies to account for the experimental resolution. Magnetic lensing effects [18] were taken into account in the simulation through the magnification or demagnification of the energy spectrum of each source. As an example we show in Fig. 2(a) the resulting distribution of $W$ for multiplets of 14 events. The significance of a given multiplet can be quantified by computing the fraction of isotropically distributed simulations, with the same total number of events as in the data and with the same energy spectrum, in which a multiplet with the same or larger multiplicity and passing the same cuts appears by chance. At high energies the UHECR angular distribution may not be isotropic, reflecting structure in the distribution of sources within the GZK horizon. However, our data set is dominated by lower energy events for which isotropy is an excellent approximation. We show in Fig. 3(a) the chance probability for multiplets of different multiplicity as a function of $W_{\text {max }}$. We note that when reducing $W_{\max }$, some of the events of the multiplets will be missed and their multiplicity will be reduced. However, the significance of a smaller multiplet passing a tighter bound on $W_{\max }$ can be larger than the significance of the complete multiplet with a looser $W_{\max }$ cut. It turns out that the largest mean significance for the simulated sources (i.e. the average of the significances of the resulting multiplets after imposing the cuts) appears when a cut $W_{\max } \simeq 1.5^{\circ}$ is applied. The angular scale of $1.5^{\circ}$ provides in fact a reasonable cut which accounts for the angular resolution and the mean value of the turbulent field deflections. We note that in the case of 14 -plets, in $50 \%$ of the simulations all the events pass this cut and the multiplet will be reconstructed as a 14 -plet, while in $38 \%$ of the cases one event is lost and in $11 \%$ of the cases two events are lost.

A similar analysis can be performed to fix the cut on the correlation coefficient $C_{\min }$. The distribution of $C(u, 1 / E)$ for the simulated 14-plets is shown in Fig. 2(b) and the chance probability for multiplets of different multiplicity as a function of $C_{\min }$ is illustrated in Fig. 3(b). The largest mean significance is attained now for values of $C_{\min }$ in the range from 0.85 to 0.9 , depending on the multiplicity considered. We will then fix in the following $W_{\max }=1.5^{\circ}$ and $C_{\min }=0.9$. Considering simulations with 14 events and for a cut $C_{\min }=0.9$, we find that in $57 \%$ of the cases all events pass the cuts, in $12 \%$ of the simulations one event is lost and in $11 \%$ of them two events are lost. We note that the choice of the optimal cuts depends slightly on the galactic magnetic field model considered in the simulations and on the modeling of the turbulent field deflections.

We will hence search for correlated multiplets of events with energies above $20 \mathrm{EeV}$ (so that the linear correlation of the deflection with $1 / E$ is still expected to be valid for proton sources) which extend up to $20^{\circ}$ in the sky (see Eq. (1)). We also require that the multiplet contains at least one event with energy above $45 \mathrm{EeV}$. We note that the energy of the most energetic event of a set of 10 events with $E>20 \mathrm{EeV}$ from a source with spectral index $s=2.5$ is larger than $45 \mathrm{EeV}$ with a probability of $97 \%$ (for a spectral index $s=3$ this probability is $\sim 90 \%$ and for $s=2$ it is $\sim 99.7 \%$ ). Hence, requiring one high energy event above $45 \mathrm{EeV}$ is not restrictive, and it simplifies the strategy to start the search for multiplets, which proceeds by looking at all possible sets of events contained in windows of $20^{\circ}$ around those high energy events. Since we are ultimately interested in multiplicities larger than 8 (see Fig. 3 in which it is apparent that for the present statistics above $20 \mathrm{EeV}$ correlated sets of smaller multiplicity are very likely to appear by 


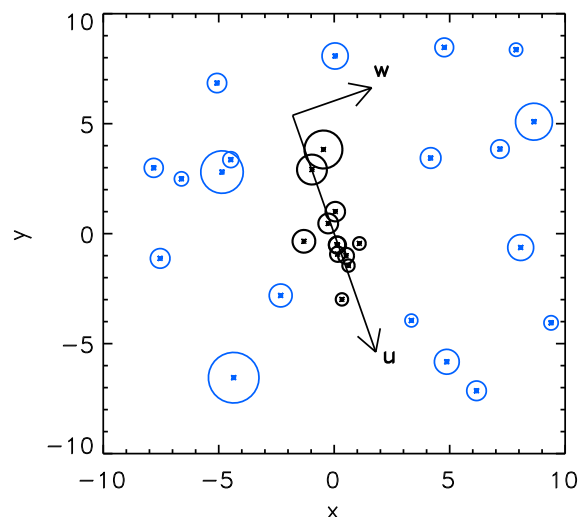

(a)

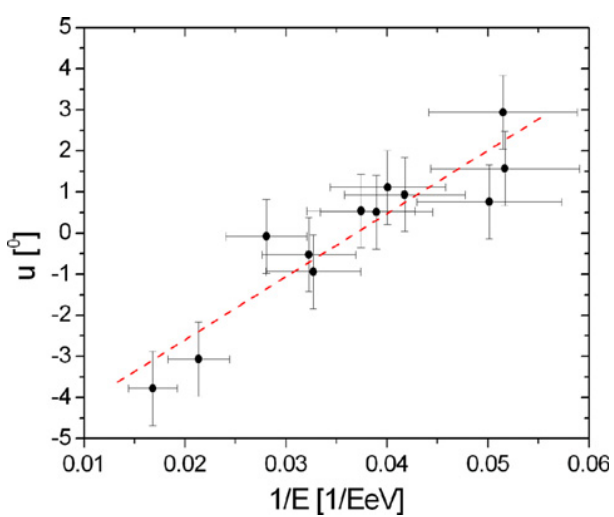

(b)

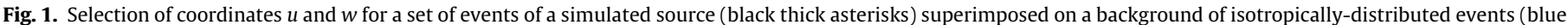

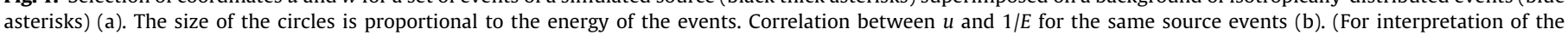
references to color in this figure legend, the reader is referred to the web version of this article.)

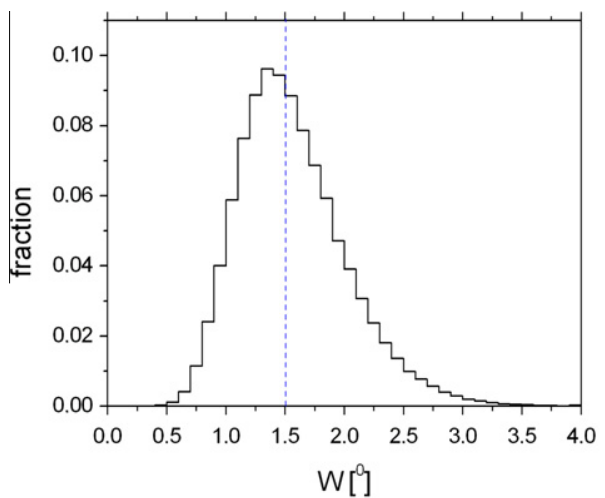

(a)

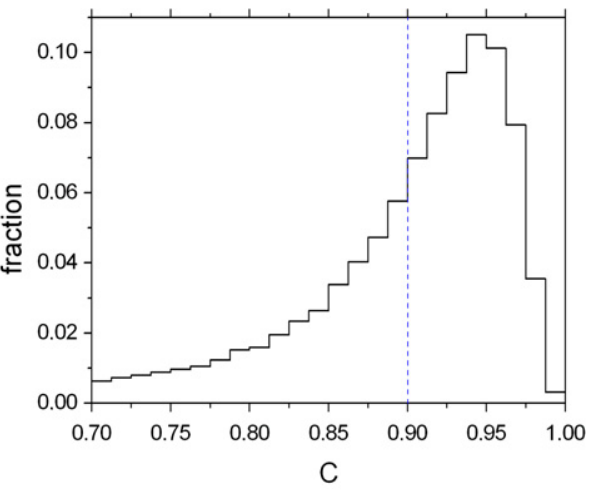

(b)

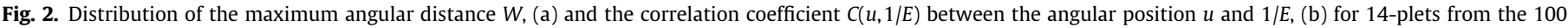
simulated sources. The vertical dashed lines indicate the cuts on $W$ and $C$ optimized for multiplicity and significance (see text).

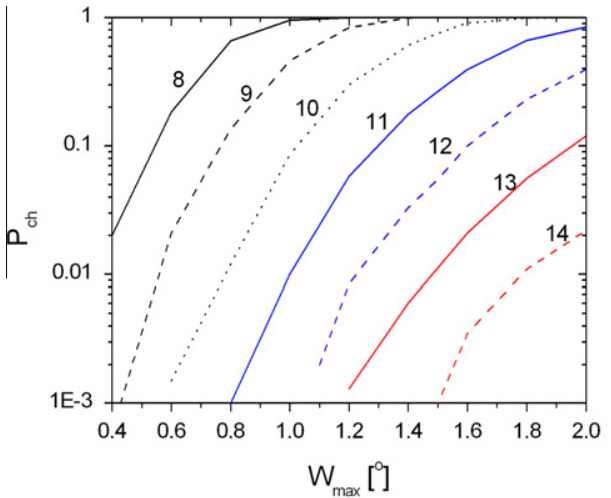

(a)

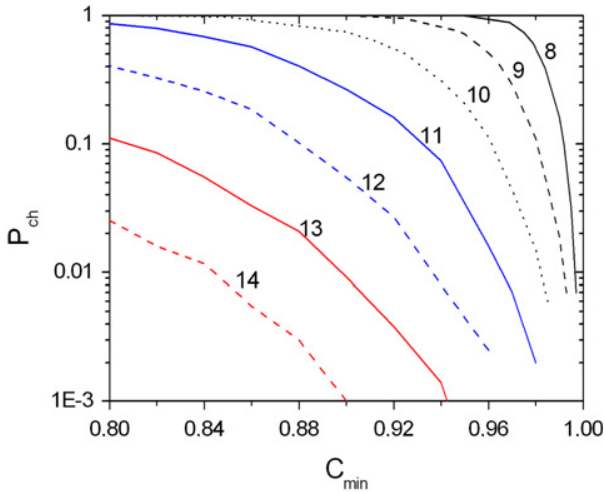

(b)

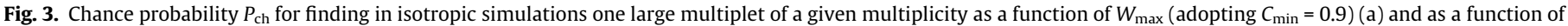
$C_{\min }\left(\right.$ adopting $\left.W_{\max }=1.5^{\circ}\right)$ (b) (see text).

chance in isotropic simulations), it is possible to make this search more efficient by first identifying the high energy end of the candidate multiplets. We hence consider for every event above $45 \mathrm{EeV}$ the quadruplets that it forms with the events within a circle of $15^{\circ}$ having energies above $25 \mathrm{EeV}$ and with a correlation coefficient $C(u, 1 / E) \geqslant 0.8$. The precise values of these cuts are not crucial as long as they allow one to safely include the larger multiplets of interest. For each of these candidates we then extend the search including all the events above $20 \mathrm{EeV}$ with an angular distance to the highest energy one smaller than $20^{\circ}$ and at a distance smaller than $3 W_{\max }$ from the quadruplet axis. This allows us to find the correlated multiplets satisfying the cuts in $W_{\max }$ and $C_{\min }$ in a very 
efficient way, as it is desirable to be able to perform a large number of simulations.

The multiplets search procedure has been designed for sources having a light composition. For sources having instead a heavy composition above $20 \mathrm{EeV}$, multiplets will be much more difficult to identify since they would typically spread through a larger region in the sky and also the linearity of their directional distribution will be lost.

Once a correlated multiplet is identified, from the linear fit to the relation

$u=u_{s}+\frac{D}{E}$

the position of the source $\left(u_{s}, 0\right)$ (in the $u-w$ coordinate system) and the deflection power $D$ can be obtained.

A true correlated multiplet arising from magnetic field deflections of events from a single source can also include by chance some events from the background that appear aligned and correlated in energy with the events from the source. We have estimated the fraction of events that is expected to be due to chance background alignments by simulating an isotropic background distribution of events with the energy of the observed events above $20 \mathrm{EeV}$ and superimposing multiplets of 12 events from simulated sources. We found that $29 \%$ of the reconstructed multiplets do not pick additional background events, while $46 \%$ just pick one additional background event and $25 \%$ pick two or more. Thus, the fraction of events added from the background is typically very small.

\section{Results}

We applied the method discussed in Section 3 to 1509 events above $20 \mathrm{EeV}$ recorded at the Pierre Auger Observatory from 1st January 2004 to 31st December 2010. We implemented a search for all possible multiplets which extend up to $20^{\circ}$ in the sky and contain at least one event with energy above $45 \mathrm{EeV}$, and that have a half-width smaller than $W_{\max }=1.5^{\circ}$ and a correlation coefficient larger than $C_{\min }=0.9$. The largest multiplet found in this data set is one 12-plet and there are also two independent decuplets. They are displayed in Fig. 4. Their deflection power, position of the potential source location and correlation coefficient are listed in Table 1. Decuplet II in Table 1 consists of three dependent sets of ten events $(a-c)$ that are formed by the combination of a set of twelve events. These three decuplets are not independent of each other since they have most events in common. The uncertainties in the reconstruction of the position of the potential sources have been

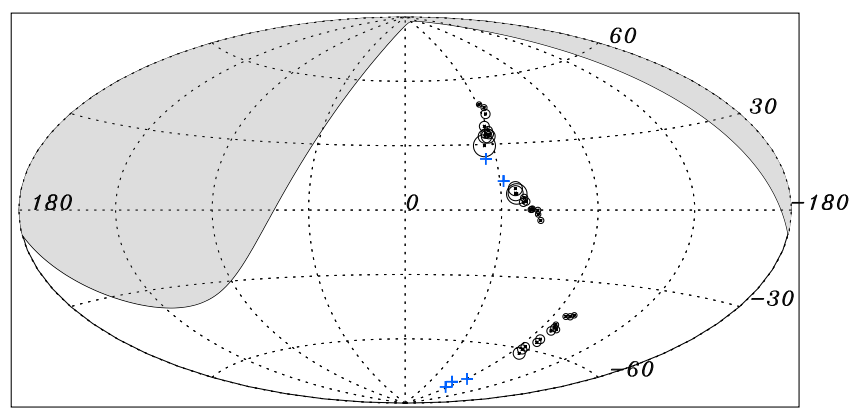

Fig. 4. Observed multiplets with 10 or more events in galactic coordinates. The size of the circles is proportional to the energy of the event. Plus signs indicate the positions of the potential sources for each multiplet. One decuplet is in fact three dependent decuplets that are formed by the combination of twelve events and the three corresponding reconstructions of the potential sources are shown. The solid line represents the border of the field of view of the Southern Observatory for zenith angles smaller than $60^{\circ}$ and the grey shaded area is the region outside the field of view.
Table 1

Deflection power, $D$; reconstructed position of the potential source in galactic coordinates, $(l, b)_{s}$; uncertainty in the reconstructed position of the potential source along the direction of deflection, $\Delta u_{s}$, and orthogonal to it, $\Delta w_{s}$; and linear correlation coefficient, $C$, for the largest correlated multiplets found. The data correspond to events with energy above $20 \mathrm{EeV}$ from 1st January 2004 to 31st December 2010.

\begin{tabular}{llllll}
\hline Multiplet & $D\left[{ }^{\circ} 100 \mathrm{EeV}\right]$ & $(l, b)_{S}\left[^{\circ}\right]$ & $\Delta u_{S}\left[^{\circ}\right]$ & $\Delta w_{S}\left[^{\circ}\right]$ & $C$ \\
\hline 12-plet & $4.3 \pm 0.7$ & $(-46.7,13.2)$ & 2.4 & 0.9 & 0.903 \\
10-plet I & $5.1 \pm 0.9$ & $(-39.9,23.4)$ & 2.7 & 0.9 & 0.901 \\
10-plet IIa & $8.2 \pm 1.3$ & $(-85.6,-80.4)$ & 4.3 & 1.9 & 0.920 \\
10-plet IIb & $7.6 \pm 1.2$ & $(-79.6,-77.9)$ & 4.0 & 1.6 & 0.919 \\
10-plet IIc & $6.5 \pm 1.1$ & $(-91.5,-75.7)$ & 3.9 & 1.6 & 0.908 \\
\hline
\end{tabular}

calculated propagating the uncertainties in energy and arrival direction to an uncertainty in the rotation angle (Eq. (5)) and in the linear fit performed to the deflection vs. 1/E (Eq. (7)).

The probability that the observed number (or more) of correlated multiplets appears by chance can be computed by applying a similar analysis to simulations of randomly distributed events weighted by the geometric exposure of the experiment [19] and with the energies of the observed events. The fraction of simulations with at least one multiplet with 12 or more events is $6 \%$, and the fraction having at least three multiplets with 10 or more events is $20 \%$. Therefore, there is no statistically significant evidence for the presence of multiplets from actual sources in the data. We note that with the present statistics, an individual multiplet passing the required selection cuts should have at least 14 correlated events in order that its chance probability be $10^{-3}$.

Measurements by the Pierre Auger Observatory [20] of the depth of shower maximum and its fluctuations indicate a trend towards heavy nuclei with increasing energy. This interpretation of the shower depths is not certain, however. It relies on shower simulations that use hadronic interaction models to extrapolate particle interaction properties two orders of magnitude in centreof-mass energy beyond the regime where they have been tested experimentally. Magnetic alignment and correlation with the inverse of the energy as searched here are not expected for heavy nuclei. Assuming there are sources which accelerate an appreciable proton component, the non-observation of significant multiplets could be the consequence of having a large density of sources. Given the present statistics, the maximum source density which would allow to observe a multiplet containing 12 events above $20 \mathrm{EeV}$ from the nearest source to the Earth can be roughly estimated by considering that this source should produce a fraction $12 / 1509 \approx 1 / 125$ of the total flux observed in the field of view of the Auger Observatory in this energy range. Assuming that the sources have equal intrinsic luminosity and are uniformly distributed and that cosmic rays in this energy range can arrive from distances up to about $1 \mathrm{Gpc}$, the above mentioned constraints imply that the nearest source should be within $\sim 10 \mathrm{Mpc}$. Thus, the mean local density of sources should not be larger than a few $10^{-4} \mathrm{Mpc}^{-3}$. The fact that we have not seen a larger multiplet is an indication that the density of sources is probably larger. This very rough estimation is subject to large fluctuations but it is indicative that densities within the current lower limits may lead to the kind of signals searched for here. We note, however, that this bound would be relaxed if contributions of heavy cosmic ray primaries become significant, or if very strong turbulent magnetic fields were present.

\section{Conclusions}

A search for ultra-high energy cosmic ray multiplets was performed in the data gathered between 1st January 2004 and 31st December 2010 by the Pierre Auger Observatory with energy 
above $20 \mathrm{EeV}$. The largest multiplet found was one 12-plet. The probability that it appears by chance from an isotropic distribution of events is $6 \%$. Thus, there is no significant evidence for the existence of correlated multiplets in the present data set. Future data will be analyzed to check if some of the observed multiplets grow significantly or if some new large multiplets appear.

\section{Acknowledgments}

The successful installation, commissioning and operation of the Pierre Auger Observatory would not have been possible without the strong commitment and effort from the technical and administrative staff in Malargüe.

We are very grateful to the following agencies and organizations for financial support: Comisión Nacional de Energía Atómica, Fundación Antorchas, Gobierno De La Provincia de Mendoza, Municipalidad de Malargüe, NDM Holdings and Valle Las Leñas, in gratitude for their continuing cooperation over land access, Argentina; the Australian Research Council; Conselho Nacional de Desenvolvimento Científico e Tecnológico (CNPq), Financiadora de Estudos e Projetos (FINEP), Fundação de Amparo à Pesquisa do Estado de Rio de Janeiro (FAPERJ), Fundação de Amparo à Pesquisa do Estado de São Paulo (FAPESP), Ministério de Ciência e Tecnologia (MCT), Brazil; AVCR AV0Z10100502 and AV0Z10100522, GAAV KJB100100904, MSMT-CR LA08016, LC527, 1M06002, and MSM0021620859, Czech Republic; Centre de Calcul IN2P3/CNRS, Centre National de la Recherche Scientifique (CNRS), Conseil Régional Ile-de-France, Département Physique Nucléaire et Corpusculaire (PNC-IN2P3/CNRS), Département Sciences de l'Univers (SDU-INSU/CNRS), France; Bundesministerium für Bildung und Forschung (BMBF), Deutsche Forschungsgemeinschaft (DFG), Finanzministerium Baden-Württemberg, Helmholtz-Gemeinschaft Deutscher Forschungszentren (HGF), Ministerium für Wissenschaft und Forschung, Nordrhein-Westfalen, Ministerium für Wissenschaft, Forschung und Kunst, Baden-Württemberg, Germany; Istituto Nazionale di Fisica Nucleare (INFN), Ministero dell'Istruzione, dell'Università e della Ricerca (MIUR), Italy; Consejo Nacional de Ciencia y Tecnología (CONACYT), Mexico; Ministerie van Onderwijs, Cultuur en Wetenschap, Nederlandse Organisatie voor Wetenschappelijk Onderzoek (NWO), Stichting voor Fundamenteel Onderzoek der Materie (FOM), Netherlands; Ministry of Science and Higher Education, Grant Nos. 1 P03 D 014 30, N202
090 31/0623, and PAP/218/2006, Poland; Fundação para a Ciência e a Tecnologia, Portugal; Ministry for Higher Education, Science, and Technology, Slovenian Research Agency, Slovenia; Comunidad de Madrid, Consejería de Educación de la Comunidad de Castilla La Mancha, FEDER funds, Ministerio de Ciencia e Innovación and Consolider-Ingenio 2010 (CPAN), Xunta de Galicia, Spain; Science and Technology Facilities Council, United Kingdom; Department of Energy, Contract Nos. DE-AC02-07CH11359, DE-FR02-04ER41300, National Science Foundation, Grant No. 0450696, The Grainger Foundation USA; ALFA-EC/ HELEN, European Union 6th Framework Program, Grant No. MEIF-CT-2005-025057, European Union 7th Framework Program, Grant No. PIEF-GA-2008-220240, and UNESCO.

\section{References}

[1] J.L. Han, IAU Symp. 259 (2009) 455. <aXiv:0901.1165[astro-ph]>.

[2] R. Beck, AIP Conf. Proc. 1085 (2009) 83. <arXiv:0810.2923[astro-ph]>.

[3] J.C. Brown, ASP Conf. Ser. 438 (2011) 216. <arXiv:1012.2932[astro-ph]>

[4] M.S. Pshirkov, P.G. Tinyakov, P.P. Kronberg, K.J. Newton-McGee, Astrophys. J. 738 (2011) 192. <arXiv:1103.0814[astro-ph]>.

[5] R.J. Rand, S.R. Kulkarni, Astrophys. J. 343 (1989) 760.

[6] H. Ohno, S. Shibata, MNRAS 262 (1993) 953.

[7] G. Sigl, F. Miniati, T. Ensslin, Phys. Rev. D 68 (2003) 043002. <arXiv:astro-ph/ 0302388>.

[8] K. Dolag, D. Grasso, V. Springel, I. Tkachev, J. Cosmology Astropart. Phys. 0501 (2005) 009. <arXiv:astro-ph/0410419>.

[9] S. Das, H. Kang, D. Ryu, J. Cho, Astrophys. J. 682 (2008) 29. <arXiv:astroph.0801.0371>.

[10] J. Abraham et al., The Pierre Auger Collaboration, Nucl. Instrum. Meth. A523 (2004) 50.

[11] I. Allekotte et al., Nucl. Instrum. Meth. A586 (2008) 409.

[12] J. Abraham et al., The Pierre Auger Collaboration, Nucl. Instrum. Meth. A620 (2010) 227

[13] J. Abraham et al., The Pierre Auger Collaboration, Nucl. Instrum. Meth. A613 (2010) 29.

[14] C. Bonifazi, for The Pierre Auger Collaboration, Nucl. Phys. B (Proc. Suppl. 19) (2009) 20.

[15] C. Di Giulio, for the Pierre Auger Collaboration, in: Proc. 31st International Cosmic Ray Conference, 2009 <arXiv:0906.2189[astro-ph]>;

R. Pesce, for the Pierre Auger Collaboration, in: Proc. 32nd International Cosmic Ray Conference, in press.

[16] G. Golup, D. Harari, S. Mollerach, E. Roulet, Astropart. Phys. 32 (2009) 269. <arXiv:0902.1742[astro-ph/]>.

[17] T. Stanev, Astrophys. J. 479 (1997) 290. <arXiv:astro-ph/9607086>.

[18] D. Harari, S. Mollerach, E. Roulet, J. High Energy Phys. 08 (1999) 022. <arXiv:astro-ph/9906309>.

[19] P. Sommers, Astropart. Phys. 14 (2001) 271. <arXiv:astro-ph/0004016>.

[20] J. Abraham et al., The Pierre Auger Collaboration, Phys. Rev. Lett. 104 (2010) 091101. 